\title{
Dark-Soliton Molecules in an Exciton-Polariton Superfluid
}

\author{
Anne Maître®, ${ }^{1, *}$ Giovanni Lerario, ${ }^{1,2}$ Adrià Medeiros $\odot,{ }^{1}$ Ferdinand Claude, ${ }^{1}$ Quentin Glorieux $\odot,{ }^{1}$ \\ Elisabeth Giacobino, ${ }^{1}$ Simon Pigeon ${ }^{1},{ }^{1}$ and Alberto Bramati ${ }^{1}$ \\ ${ }^{1}$ Laboratoire Kastler Brossel, Sorbonne Université, CNRS, ENS-Université PSL, \\ Collège de France, Paris 75005, France \\ ${ }^{2}$ CNR NANOTEC, Istituto di Nanotecnologia, via Monteroni, 73100 Lecce, Italy
}

(Received 28 February 2020; revised 27 August 2020; accepted 28 September 2020; published 9 November 2020)

\begin{abstract}
The general theory of dark solitons relies on repulsive interactions and, therefore, predicts the impossibility to form dark-soliton bound states. One important exception to this prediction is the observation of bound solitons in nonlocal nonlinear media. Here, we report that exciton-polariton superfluids can also sustain darksoliton molecules, although the interactions are fully local. With a novel all-optical technique, we create two dark solitons that bind together to form an unconventional dark-soliton molecule. We demonstrate that the stability of this structure and the separation distance between two dark solitons is tightly connected to the driven-dissipative nature of the polariton fluid.
\end{abstract}

DOI: 10.1103/PhysRevX.10.041028

Subject Areas: Fluid Dynamics, Nonlinear Dynamics, Optics

\section{INTRODUCTION}

Solitons are localized waves propagating with unchanged shape in a nonlinear medium. Depending on the nature of the nonlinearity, focusing or defocusing, solitons can be either bright, with a localized bump, or dark, with a dip on a homogeneous background. While the bright solitons can attract or repel each other depending on their relative phase [1], the dark solitons are known to experience only mutual repulsive forces [2-5].

Dark solitons are observed in several nonlinear systems, such as cold atom condensates [3,6,7], thin magnetic films $[8,9]$, liquid helium [10], optical fibers [11,12], and more recently on the surface of water [13]. Differently from bright solitons which might attract and form molecules [14], due to repulsive interactions, dark-soliton bound states cannot form in nonlinear local media, unless specific stabilization schemes are applied in multicomponent vector solitons [15].

Interestingly, in nonlinear media with nonlocal interactions, an attractive behavior between dark solitons has been predicted $[16,17]$ and experimentally observed in a thermo-optic medium [18]. In this paper, we present experimental and numerical results in striking contrast with these predictions. We demonstrate the creation and

*anne.maitre@lkb.upmc.fr

Published by the American Physical Society under the terms of the Creative Commons Attribution 4.0 International license. Further distribution of this work must maintain attribution to the author(s) and the published article's title, journal citation, and DOI. binding of dark solitons, forming a dark-soliton molecule [19] in a purely local medium: a quantum fluid of exciton polaritons, the half-light half-matter bosonic quasiparticles arising from the strong coupling between excitons and photons in semiconductor microcavities. Indeed, the polariton-polariton interaction is dominated by the electronelectron and hole-hole exchange interaction of their excitonic components, which is fully local and is modeled by a contact interaction [20]. This unconventional behavior for dark solitons strongly deviates from equilibrium atomic quantum fluids as well as from previous studies in polariton fluids, where repulsive dark solitons [21-23] and halfsolitons $[24,25]$ are predicted and observed.

Despite some interesting theoretical proposals to create dark solitons in incoherently pumped polariton condensates $[26,27]$, their experimental observation is still missing in this configuration due to the limited stability theoretically demonstrated in Ref. [28]. On the contrary, here, we use a novel all-optical imprinting method which exploits the specific features of the resonant pumping and of the bistable regime exhibited by the system. We observe a highly stable dark-soliton molecule, which propagates on a polariton fluid for macroscopic distances. Remarkably, the intrasoliton distance is independent of the properties of the fluid and is determined only by the intrinsic dissipation of the polariton system.

The importance of our work is twofold: On one hand, for the first time, we observe a stable dark-soliton molecule in a nonlinear medium with repulsive local interactions, a result which represents a significant advance in the general understanding of nonlinear phenomena; on the other hand, the all-optical, widely tunable technique of our experiment 
is fully scalable and enables the creation of stable multiple soliton patterns.

Moreover, we anticipate that this technique will be widely exploited for the systematic study of quantum turbulence. Indeed, while in the present work we manage to generate stable solitons in supersonic flows [29], the imprinting technique and the full control of the fluid velocity easily allow one to imprint dark solitons on polariton fluids with deeply subsonic velocities. In these conditions, dark solitons are unstable due to the onset of the snake instabilities and break into quantum vortexantivortex pairs, giving rise to a quantum analog of the classical von Kármán vortex street [30]. Therefore, the imprinting technique could give significant contributions to elucidate open questions as the formation of turbulent cascades in the presence of dissipation at all length scales [31], as in polaritons, and the validity of the entropy arguments that explain the formation of inverse cascades in equilibrium two-dimensional systems [32-34].

Finally, the imprinting technique should be straightforwardly transferable to the wide class of quantum fluids of light [35], providing a very flexible tool for the study of nonlinear phenomena in these systems.

\section{MICROCAVITY POLARITON AND BISTABILITY ENHANCEMENT OF THEIR FREE PROPAGATION}

In semiconductor microcavities, the strong coupling between quantum well excitons and cavity photons gives rise to two kinds of quasiparticles with distinct energies: the upper and lower polaritons. They combine properties from light and matter, which makes them behave like weakly interacting composite bosons. Their photonic part gives them a light effective mass and the possibility to be excited and detected optically, while their excitonic part is responsible for their interactions. The resonant pumping of exciton polaritons consists in injecting photons into the system at a specific energy and in-plane wave vector of the lower polariton branch. At high driving intensity, it results in the creation of a polariton fluid whose phase coincides with the pump one. In this strongly driven regime, superfluid effects can be studied, like viscus-free flow [36]. However, the presence of the driving field prevents the free evolution of the fluid properties [37]. To overcome this constraint, spatial or temporal engineering of the excitation beam is used, leading to the observation of quantized vortices or dark-soliton pairs [21,37-41] in the driving-free region, but with a propagation distance limited by the short lifetime of the polaritons.

The introduction of a small positive energy detuning between the driving field and the lower polariton branch induces an optical bistability [42], offering two stable states for the system [Fig. 1(a)]: a linear regime with a low polariton density and negligible interactions between particles, while the high-density regime presents strong

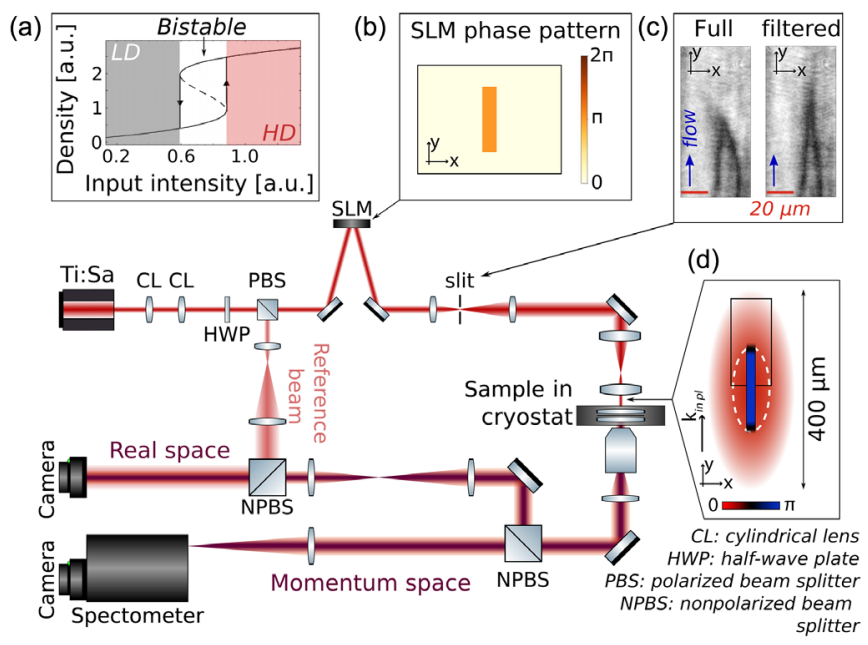

FIG. 1. Experimental setup. A Ti:sapphire laser beam with engineered shape and phase is focused on the microcavity sample with a small incidence angle to create a polariton fluid that propagates in the $y$ direction. (a) shows the theoretical bistability curve resulting from the quasiresonant pumping. The gray region corresponds to the low-density regime (LD), while the red one has much higher polariton density (HD). The white part delimits the bistable hysteresis cycle. The phase profile of the excitation beam is customized with the SLM, as plotted in (b). The beam is then spatially filtered to smooth the phase jump: (c) illustrates how the opening of the filtering slit oriented along the $x$ direction influences the soliton pair propagation in the polariton fluid. (d) shows the shape of the pump: The white dashed line corresponds to the upper bistability frontier, while the black rectangle indicates the detection field of view. The blue area inside the high-intensity region indicates where the phase is shifted by $\pi$. The smoothing of the phase jump in the $y$ direction due to the spatial filtering is indicated by the color scale. The real space image gives access to the density and phase maps (interfering with a reference beam), whereas the conditions of the driving field are extracted from the momentum space detection.

interactions and corresponds to the superfluid regime [43]. The system exhibits a hysteresis cycle, i.e., a range of input intensities where the two states are accessible, called the bistable regime in the following and highlighted in white in Fig. 1(a).

Recently, a theoretical proposal by Pigeon and Bramati [44] suggested to exploit the optical bistability to compensate the system losses without inhibiting the fluid spontaneous dynamic. Supporting the fluid with an intensity within the bistable regime releases the constraint imposed by the quasiresonant driving field and makes possible the spontaneous formation of turbulence sustained for macroscopic propagation distances $[45,46]$.

The possibility to have stationary states exhibiting phase mismatch between the driving and the fluid fields is a specific feature of the optical bistability. Indeed, when the pumping intensity is in the bistable range, the system can switch between to two competing states with different phases, avoiding the effect of locking of the phase state by 
the driving field. While this behavior is experimentally verified and numerically reproduced by integrating the Gross-Pitaevskii equation which describes the system, an analytical demonstration would require a full quantum treatment which is not available presently in the literature.

Here, we present an original method where the solitons are directly imprinted with the use of a spatial light modulator (SLM). Combining beam-shaping techniques for direct imprinting and the bistable support mechanism [44] for a long propagation length, we report the controlled generation of dark-soliton bound states and their free propagation over macroscopic distances. While the use of the bistable regime allows achieving parallel solitons also spontaneously when the fluid hits a structural defect, as shown in Ref. [46], the imprinting method has two main advantages: It allows one to finely tune the Mach number of the imprinted solitons, and it is scalable. Both these features are of paramount importance to study the onset of the turbulence in this system.

\section{EXPERIMENTAL SETUP}

The sample used in the present work is a planar microcavity which contains three $\mathrm{In}_{0.04} \mathrm{Ga}_{0.96}$ As quantum wells at the antinodes of the cavity electromagnetic field. The GaAs cavity has a length of $2 \lambda / n_{c}$, with $\lambda=835 \mathrm{~nm}$ the excitonic resonance and $n_{c}=3.54$ the cavity GaAs substrate optical index; the two Bragg mirrors consist of 21 and 24 GaAs-AlAs pairs, respectively. The linewidth of the cavity is $0.07 \mathrm{meV}$, while the Rabi splitting is $5.1 \mathrm{meV}$.

The polariton fluid is obtained by quasiresonant pumping using a continuous wave single-mode Ti:sapphire laser at a frequency slightly blue detuned from the lower polariton branch resonance (typically, an energy detuning $\Delta E=0.20 \mathrm{meV}$ ) which illuminates the cavity with a small incidence angle.

In order to imprint a particular phase profile on the fluid, one needs to excite the system above the bistability regime to fix the polariton phase properties to the pump ones. On the other hand, a free propagation of collective excitations requires a bistable fluid, in which the phase is free to evolve. Therefore, the implementation of such an imprinting mechanism requires an intensity gradient, providing a region of high intensity where the phase pattern is imprinted into the fluid, and a region of lower intensity, bistable, where the driving phase is flat and where the imprinted modulations are able to propagate freely.

This configuration is reached by using the Gaussian shape of the exciting beam [see Fig. 1(d)]. By adjusting the intensity of the pump, the central part of the illuminated area is placed above the bistability limit, i.e., in the red part of Fig. 1(a), imprinting its phase to the fluid. The phase modulation is sent only in this region, colored in blue in Fig. 1(d). The region where the local pump intensity is above the bistability cycle is delimited by the white dashed line: Outside this region, the pump has a lower intensity and induces a bistable fluid, whose phase is free to evolve. The detection field of view (black rectangle) is placed in order to observe the propagation of the imprinted excitation. The exciting beam on the sample has a vertical dimension of $400 \mu \mathrm{m}$, which induces an intensity difference of more than $10 \%$ between the center of the spot and the region $150 \mu \mathrm{m}$ above it.

\section{ALL-OPTICAL IMPRINTING OF PARALLEL DARK SOLITONS}

The phase pattern [Fig. 1(b)] is imprinted on the pump beam using an SLM, whose surface is imaged onto the sample. This process allows for a direct control of its shape and position, so that the phase jump can be placed at the limit of the bistable regime of the fluid. The extension of the high-density region and the separation of the imprinting area from the bistable region are crucial parameters to achieve the generation of soliton pairs and ensure their efficient propagation. Indeed, the imprinting area is carefully designed to be in the high-density region and to extend until its boundary in order to ensure a smooth connection to the bistable region. To this end, we design a rectangular shape where the phase is $\pi$ shifted with respect to the outer beam [Fig. 1(b)]. A slit oriented along the $x$ direction realizes a spatial filtering in the $y$ direction of the Fourier plane before imaging the SLM plane onto the sample: High spatial frequencies components are cut in the direction of the flow in order to smooth the phase jump experienced by the flow at the end of the phase pattern. The cutoff directly influences the shape of the top part of the solitons imprinted on the fluid, as a smoothing of the phase modulation induces an elongation of the solitons, illustrated in Fig. 1(c). When the slit is fully opened, the phase jump along the direction of the flow is sharp, leading to a pair of imprinted solitons closing on a short distance. However, when the slit aperture is reduced, the imprinted solitons extend along the flow, gradually approaching each other.

To be sustained by the fluid, the solitons need supersonic conditions [21,29]: Those are achieved by choosing the appropriate wave vector and energy detuning of the driving field. The sound velocity $c_{s}$ depends on the energy detuning $\Delta E$ between the laser and the lower polariton branch: $c_{s}=\sqrt{\left(g n / m^{*}\right)} \approx \sqrt{\left(\Delta E / m^{*}\right)}$, with $m^{*}$ the polariton effective mass, $g n$ the interaction energy, which is the product of the interaction constant $g$ by the polariton density $n$, and $\Delta E$ the detuning between the laser and the lower polariton branch, which is equal to $g n$ (within the polariton linewidth) in our resonant configuration. The speed of the fluid is linked to the in-plane wave vector, as it is the derivative of the dispersion: $v_{f}=\left[\partial \omega_{L P}\left(\mathbf{k}_{\|}\right) / \partial \mathbf{k}_{\|}\right]$ with $\mathbf{k}_{\|}$the driving field in-plane wave vector.

In Fig. 2(a), we illustrate the crucial role of the bistable regime with respect to the soliton behavior. The polariton flow is from bottom to top, and the red highlighted areas 
(a)

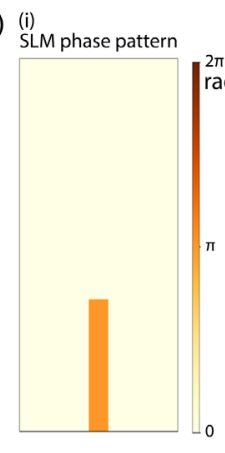

(ii)

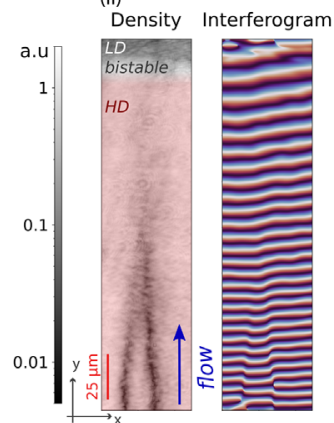

(iii)

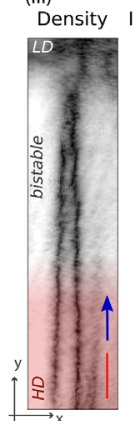

(b) (i)

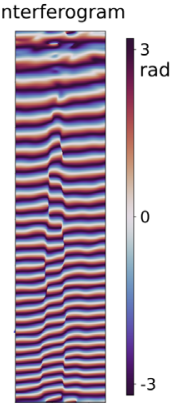

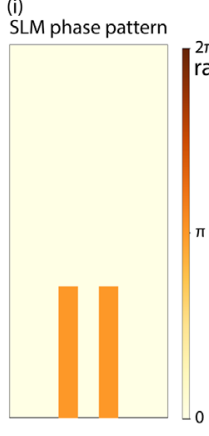

(ii)

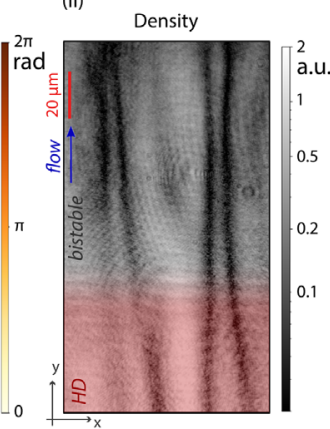

(iii)

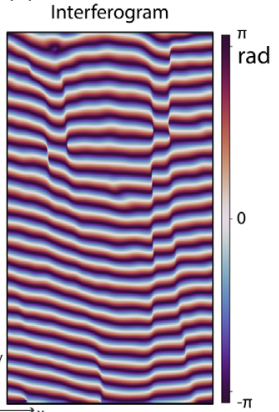

FIG. 2. Impression of parallel dark solitons. (a) Propagation of two parallel dark solitons. (i) Phase pattern created by the SLM, with the same field of view as the detection. The phase modulation is sent only on the bottom part of the image. (ii) Density (logarithmic scale) and phase at high power, where almost all the pumped area is above the bistability range and, therefore, gets the phase profile corresponding to the driving one. The top of the picture corresponds to the limit of the excitation: One can see a thin bistable region just before the density of polariton abruptly drops to the low-density regime. (ii) At lower intensity: The white area corresponds to the bistable part of the fluid where the solitons propagate freely, until they reach the lower part of the bistability range. The energy detuning $\Delta E$ is $0.13 \mathrm{meV}$, the fluid velocity is $1.35 \mu \mathrm{m} / \mathrm{ps}, c_{s}=0.31 \mu \mathrm{m} / \mathrm{ps}$, and $m^{*}=2.20 \times 10^{-34} \mathrm{~kg}$. (b) Impression of two pairs of dark solitons. (i) Phase pattern designed by the SLM, with the same field of view as the detection. (ii) Density (logarithmic scale) and (iii) phase of the four solitons propagating through the bistable part of the fluid.

indicate the regions of the fluid above the bistability cycle, where the phase is fixed by the driving field. Figure 2[a(i)] shows the phase profile created by the SLM, with the same field of view as the detection images: The phase modulation is sent only in the bottom part. In Fig. 2[a(ii)], we use a high laser intensity so that the main part of the pumped region reproduces the pump pattern: The density and phase modulation are indeed visible only in the bottom part of the picture. The interferograms are obtained using homodyne interferometry with a reference beam, extracted from the pump beam before any shaping through the SLM. The interference images are then Fourier filtered to enhance the phase pattern. The high-power images (Fig. 2[a(ii)]) are used as a reference to locate the position of the imprinted solitons. Lowering gradually the intensity of the driving field, the extension of the high-density region of the fluid is decreased; consequently, the bistable region expands, occupying an area closer and closer to the center of the driving beam and eventually reaching the imprinted solitons. The phase of the fluid readjusts, and the soliton pair propagates with the flow as long as the fluid is in the bistable region (Fig. 2[a(iii)]), despite the phase mismatch with the driving field. We can observe that the solitons remain dark with a phase jump of $\pi$ and parallel, as long as they are sustained by the fluid. As they propagate on the outer part of the excitation spot, the intensity of the pump is slowly decreasing until it reaches the threshold of the lower part of the bistability loop; the fluid then jumps to the low-density regime, where the nonlinear interactions are negligible and no solitons can be sustained anymore.

The imprinting technique is fully scalable and allows one to generate stable patterns of multiple dark solitons, by generating with the SLM a periodic arrangement of alternating phase slips and using it to excite the microcavity sample. For instance, Fig. 2(b) presents the imprinting of two pairs of dark solitons. Figure 2[b(i)] illustrates the top part of the SLM phase pattern corresponding to the detection field of view: Four solitons are created equidistantly. Figure $2[b(i i)]$ shows the fluid density in logarithmic scale, with the imprinting region in red and a flow from bottom to top, and Fig. 2[b(iii)] the corresponding interferogram with a propagation of the phase modulation due to the solitons.

\section{DARK-SOLITON BOUND STATES}

The parallel propagation of the solitons assures them to stay dark and maintain their phase jump. However, this configuration is not exclusive: One can find some situations where the solitons reflect on each other, leading them to open and vanish along their propagation (see Fig. 3). In that case, they become gray solitons, as their phase jump is not $\pi$ anymore, and can be described as Bloch domain walls. In particular, a sensitive parameter is the spatial filtering of the phase jump described previously, as shown in Fig. 3. The opening of the slit in the Fourier plane of the SLM directly influences the shape of the solitons in the fluid: In Fig. 3(a), the slit is open and the phase jump is well defined when entering the cavity; it blurs on the fluid only because of the flow. In this case, the solitons do not propagate in the bistable region. From Fig. 3(b) to Fig. 3(d), the slit is gradually closed, leading to a higher spatial filtering and a longer propagation of the solitons. Yet, when the angle between the two solitons is too high, they bounce on each other, reflect, and vanish along their propagation. They reflect with the same angle with which they meet, respectively, $25^{\circ}, 17^{\circ}$, and $13^{\circ}$ for Fig. 3(b), 3(c), and 3(d). Finally, in Fig. 3(e), they meet with a very small angle $\left(11^{\circ}\right)$ and 


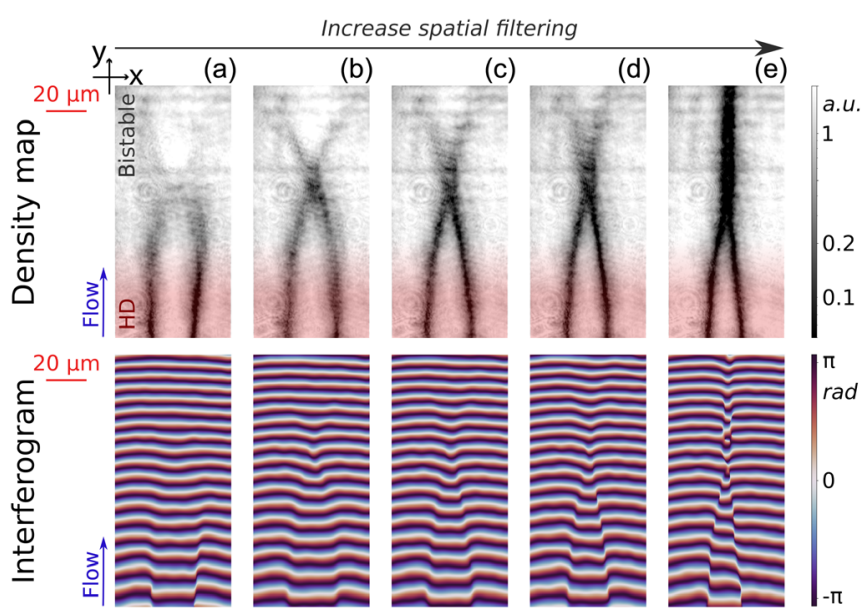

FIG. 3. Set of solitons impression with different spatial filtering. In (a), the phase jump is not filtered: On the fluid, it is blurred by the flow, but the solitons do not propagate on the bistable region. From (b) to (d), the filtering of the spatial components is gradually increased. The solitons start to propagate, but the angles between them remain too high and they bounce on each other, vanishing along their propagation. In (e), the spatial components are filtered enough so that they can align and propagate parallel, remaining dark solitons. For this set, the detuning between the laser and the lower polariton branch $\Delta E$ is $0.11 \mathrm{meV}$, the flow speed is $v_{f}=0.66 \mu \mathrm{m} / \mathrm{ps}$, the speed of sound $c_{s}=0.42 \mu \mathrm{m} / \mathrm{ps}$, and the effective polariton mass $m^{*}=1.04 \times 10^{-34} \mathrm{~kg}$.

align, propagating parallel for a hundred microns. The pictures of Fig. 3 correspond to a fluid velocity $v_{f}=0.66 \mu \mathrm{m} / \mathrm{ps}$, a speed of sound $c_{s}=0.42 \mu \mathrm{m} / \mathrm{ps}$, an effective polariton mass $m^{*}=1.04 \times 10^{-34} \mathrm{~kg}$, and an energy detuning $\Delta E=0.11 \mathrm{meV}$. The flow is from bottom to top, and the imprinting is done in the red region.

In order to see the effect of the soliton-soliton interactions, we tune the imprinted separation distance, and we observe that the freely propagating solitons tend to get closer to one another, before aligning at a specific distance, independently of the initial one. Figure 4(a) shows a set of measurements where the distance between the imprinted solitons varies: from $24 \mu \mathrm{m}$ for Fig. $4[\mathrm{a}(\mathrm{i})]$ to $15 \mu \mathrm{m}$ for Fig. $4[\mathrm{a}(\mathrm{v})]$. The fluid flow is still from bottom to top and the solitons imprinted in the red highlighted region. The fluid velocity is $1.00 \mu \mathrm{m} / \mathrm{ps}, c_{s}=0.67 \mu \mathrm{m} / \mathrm{ps}$, the effective polariton mass $m^{*}=1.24 \times 10^{-34} \mathrm{~kg}$, and the energy detuning $\Delta E=0.34 \mathrm{meV}$. Along their propagation, they get closer to one another, until reaching an equilibrium separation distance of about $5 \mu \mathrm{m}$, at which they align and propagate parallel for more than $50 \mu \mathrm{m}$. It is remarkable that the phase jump along the soliton line remains close to its imprinted value $\pi$. To reproduce the soliton behavior observed in the experiments, we perform numerical simulations based on a split-step method considering the exciton-cavity photon basis [37]. The coupled equations for the excitons $\left(\psi_{X}\right)$ and cavity photon $\left(\psi_{\mathrm{ph}}\right)$ fields are
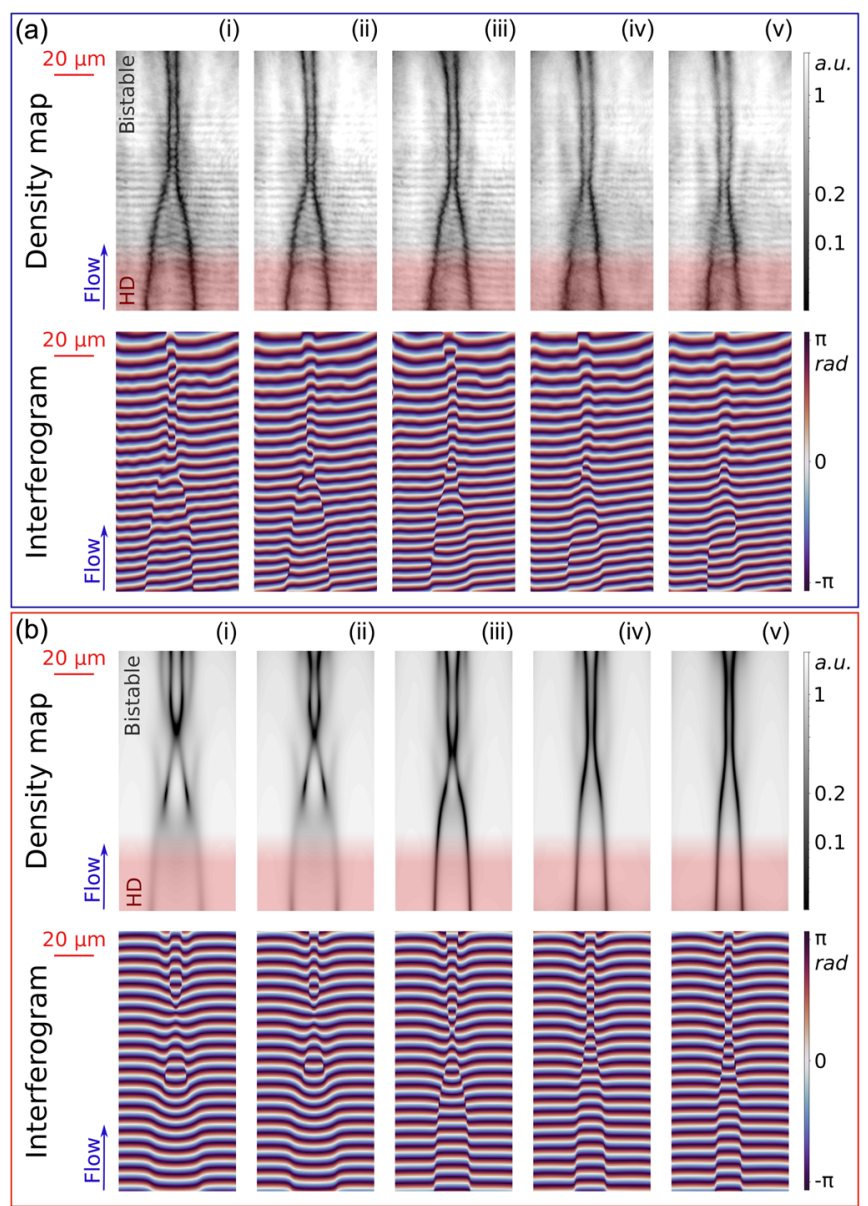

FIG. 4. Set of solitons aligning to each other for different imprinting distances. The red area indicates the imprinting region of the fluid. The fluid flow is from bottom to top. (a) Experimental data, with the density map on top and the corresponding phase map below. Solitons get closer together along their propagation, until reaching a minimal distance from which they align parallel. For this set, the energy detuning $\Delta E$ is $0.34 \mathrm{meV}$, for a fluid velocity of $1.00 \mu \mathrm{m} / \mathrm{ps}$, a speed of sound of $0.67 \mu \mathrm{m} / \mathrm{ps}$, and an effective polariton mass of $m^{*}=1.24 \times 10^{-34} \mathrm{~kg}$. (b) Numerical simulations which show the same behavior: As soon as the driving intensity is reduced enough to reach the bistable regime, imprinted solitons enter a transition region where they get closer, until a minimal distance from which they align.

$\begin{aligned} i \hbar \frac{\partial \psi_{\mathrm{ph}}}{\partial t} & =\left[-\frac{\hbar^{2} \nabla^{2}}{2 m}-i \Gamma_{\mathrm{ph}}\right] \psi_{\mathrm{ph}}+\hbar \Omega_{R} \psi_{X}+F(\mathbf{r}) e^{-i \omega_{0} t}, \\ i \hbar \frac{\partial \psi_{X}}{\partial t} & =\left[g_{X}\left|\psi_{X}\right|^{2}-i \Gamma_{X}-\Delta E_{X}\right] \psi_{X}+\hbar \Omega_{R} \psi_{\mathrm{ph}} .\end{aligned}$

The parameters (cavity photon mass $m$, laser energy $\omega_{0}$, photon decay rate $\Gamma_{\mathrm{ph}}$, half-Rabi splitting $\Omega_{R}$, and cavityexciton detuning $\Delta E_{X}$ ) are taken to be the same as in the experiment. The exciton lifetime $\tau_{X}=1 /\left(2 \pi \Gamma_{X}\right)$ is taken to be 150 ps. $F(\mathbf{r})$ describes the spatial amplitude and phase profile of the resonant pump. The numerical simulations are presented in Fig. 4(b). The driving profile corresponds to a 
large Gaussian beam centered on the lower part of the image. It creates a slightly decreasing driving intensity from bottom to top. In the high-intensity region (in red in Fig. 4), a $\pi$ phase jump is imprinted, and the effect of the slit is reproduced smoothing the phase jump in the direction of the flow, according to the experiment. The density and interferogram patterns presented correspond to steady state solutions. They reproduce accurately the experimental results, with an alignment of the solitons at a specific distance of about $5 \mu \mathrm{m}$ independently of their initial ones.

This convergence to an equilibrium configuration is analyzed in Fig. 5(a). We show how the distance between the solitons evolves along their propagation, for different initial separation distances, identified by the different color lines. After a transition region where the solitons get closer to one another, they all reach the same value, of $d_{\text {sep }}=4.8 \mu \mathrm{m}$ in this case. The separation distances are extracted from the images by fitting the transverse soliton profile, plotted in the inset in Fig. 5(a): $|\psi(x)|^{2}=$ $\tanh ^{2}\left[\left(x-d_{\text {sep }} / 2\right) / A\right] \tanh ^{2}\left[\left(x+d_{\text {sep }} / 2\right) / A\right] \quad$ [47], where $A$ corresponds to the full width at half maximum of the solitons.

\section{DRIVEN-DISSIPATIVE STABILIZATION OF DARK-SOLITON MOLECULES}

This parallel alignment is in striking contrast with the conventional behavior of dark solitons. Far from repelling each other as theoretically expected for Bose-Einstein condensates [48] and observed in polariton fluids without support [21], in the present situation, they get closer to one another before reaching an equilibrium position. This equilibrium dynamically occurs during the establishment of the steady state, due to the propagation of the domain walls separating the regions in the upper and lower bistability branches [30]. Depending on their velocities, these domains can either spread away or propagate toward the center, inducing the switch of the whole illuminated area to the upper bistability branch. For a well-adjusted range of the parameters, however, the domain wall velocity toward the center is weak and can be stabilized by the repulsive interaction between the two nonlinear shock waves associated with the domain walls.

In this case, the solitons approach each other until they reach a fixed distance for which the driving pressure and their repulsive interaction equilibrate.

The parallel alignment of the solitons and their separation distance should be very sensitive to the hydrodynamic conditions of the fluid. In particular, the characteristic length of the system, the healing length $\xi=\left(\hbar / \sqrt{2 m^{*} \Delta E}\right)$, which for the experimental conditions of the set presented in Fig. 4 is $1.17 \mu \mathrm{m}$, is expected to play a crucial role by setting the minimal achievable separation distance. As mentioned above in our resonant configuration, the detuning $\Delta E$ between the laser and the lower polariton branch is (a)

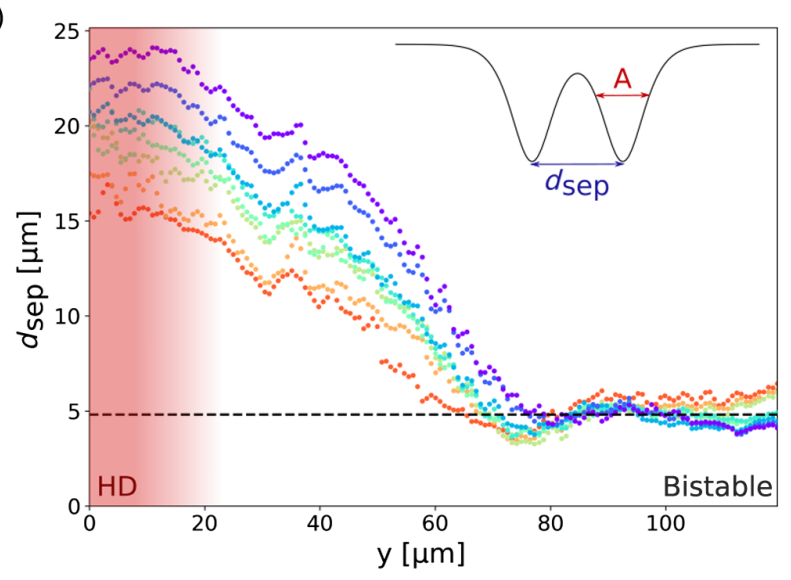

(b)

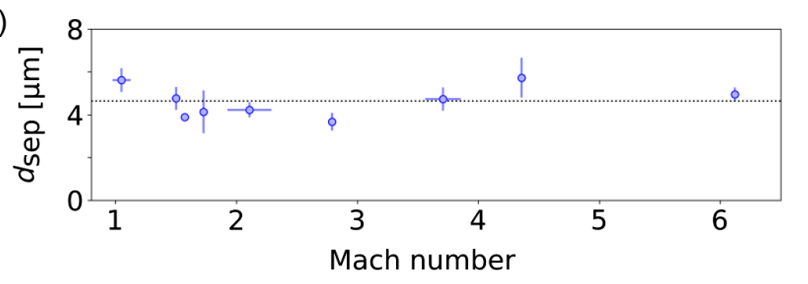

(c)

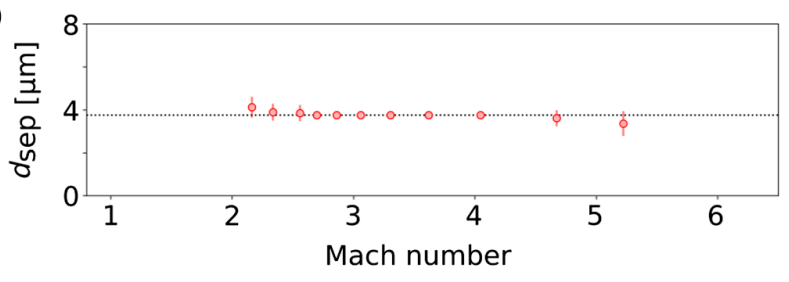

FIG. 5. (a) Evolution of the distance between the solitons along their propagation for different initial imprinting distances. Each color line corresponds to one picture with a different imprinted separation distance. Solitons tend to align at an equilibrium distance from each other. The energy shift $\Delta E=$ $0.34 \mathrm{meV}$, the fluid velocity is $1.00 \mu \mathrm{m} / \mathrm{ps}, c_{s}=0.67 \mu \mathrm{m} / \mathrm{ps}$, and $m^{*}=1.24 \times 10^{-34} \mathrm{~kg}$. The black dashed line illustrates the equilibrium separation distance at $4.8 \mu \mathrm{m}$. The distances are extracted from the fit of the transverse soliton profile, shown in the inset. (b) Summary of the experimental equilibrium separation distances for different hydrodynamic conditions, as a function of the Mach number. It stays constant around $4.75 \mu \mathrm{m}$. The parameters of each set are listed in Table I. (c) Numerical simulations done by tuning only the energy shift $\Delta E$ and keeping constant the fluid velocity and the effective mass as the experimental ones.

equal to the product $g n$ of the interaction constant $g$ and the polariton density $n$. The experiment is therefore performed in different hydrodynamic conditions in order to check whether there is a correlation between the healing length and the equilibrium distance between the solitons. The detuning $\Delta E$ between the laser and the lower polariton branch can easily be changed; however, it induces a change of the bistability cycle and, therefore, perturbs the alignment of the solitons. This perturbation can be compensated by changing the in-plane wave vector-and, thus, the speed of the fluid - to find another configuration favorable to the 
TABLE I. Parameters of the experimental sets plotted in Fig. 5(b).

\begin{tabular}{lcccc}
\hline \hline$\Delta E[\mathrm{meV}]$ & $c_{s}[\mu \mathrm{m} / \mathrm{ps}]$ & $m^{*}[\mathrm{~kg}]$ & $v_{f}[\mu \mathrm{m} / \mathrm{ps}]$ & $M$ \\
\hline 0.24 & 0.60 & $1.05 \times 10^{-34}$ & 0.62 & 1.05 \\
0.34 & 0.67 & $1.24 \times 10^{-34}$ & 1.00 & 1.50 \\
0.11 & 0.42 & $1.04 \times 10^{-34}$ & 0.66 & 1.57 \\
0.33 & 0.60 & $1.47 \times 10^{-34}$ & 1.03 & 1.73 \\
0.08 & 0.33 & $1.11 \times 10^{-34}$ & 0.70 & 2.11 \\
0.22 & 0.42 & $1.95 \times 10^{-34}$ & 1.17 & 2.79 \\
0.16 & 0.39 & $1.75 \times 10^{-34}$ & 1.43 & 3.71 \\
0.07 & 0.31 & $1.25 \times 10^{-34}$ & 1.33 & 4.36 \\
0.06 & 0.23 & $1.84 \times 10^{-34}$ & 1.42 & 6.12 \\
\hline \hline
\end{tabular}

soliton parallel propagation. The effective polariton mass $m^{*}$ depends on the detuning between the bare exciton energy and the cavity photon one: It can be tuned experimentally by changing the working point on the sample, since it has a small wedge between the two Bragg mirrors. Several sets of experiments are realized; the parameters are listed in Table I, and the results are summarized in Fig. 5(b). Each point corresponds to the average equilibrium distance of a set of free propagating solitons, in the same hydrodynamic conditions but imprinted with different initial separation distances. The equilibrium distances obtained for each set of measurements are plotted here as a function of the Mach number $M=v_{f} / c_{s}$, which is proportional to the healing length $\xi$ for constant $m^{*}$ and $v_{f}$. Quite surprisingly, no significant changes appear in the equilibrium separation distance of the solitons, no matter the considered experimental conditions. The equilibrium distance is, therefore, independent from the healing length of the fluid.

As previously explained, experimentally, those parameters can hardly be studied independently; however, numerically, they can be separately tuned. Figure 5(c) presents the calculated separation distance of the soliton pairs as a function of the Mach number. In order to specifically investigate the correlation with the healing length, in the simulations, only the detuning between the pump and the lower polariton branch $\Delta E$ is changed; the effective mass and the speed of the fluid are kept constant. The range of accessible Mach numbers is, therefore, smaller than the one observed in the experiments. However, we clearly see that the separation distance is mostly unaffected by the change of the Mach number. The error bars come from the small breathing of the soliton pair, similar to what is predicted in Refs. [17,30]. Such effects are too small to be accurately resolved experimentally in the present conditions. From Figs. 5(b) and 5(c), we can conclude that the separation distance is independent of the fluid hydrodynamics properties.

If the separation distance is disconnected from the fluid properties, then remains only the driven-dissipative nature of the fluid. In order to better understand which parameters

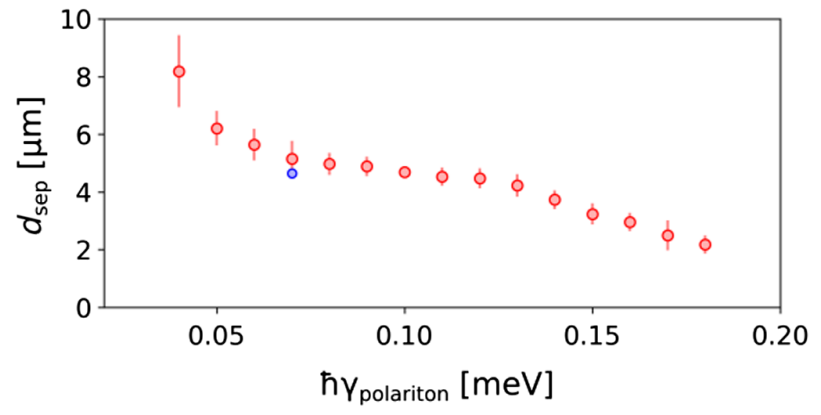

FIG. 6. Numerical simulations of the equilibrium separation distance as a function of the decay rate of the polariton (red dots). The blue dot corresponds to the experimental value for our sample. The error bars result from fluctuation of the solitons in the region where the distance is averaged.

control the equilibrium distance, we numerically investigate the influence of the decay rate of the polaritons $\gamma_{\text {polariton }}$, plotted in Fig. 6. In the simulations, the speed of sound $c_{s}$ and the speed of the fluid $v_{f}$ are kept constant and correspond to the typical experimental ones; only the polariton decay rate changes. Figure 6 clearly shows that the lifetime of the polariton plays a central role in fixing the equilibrium distance of the solitons: The more dissipation, the closer the solitons align to each other. Indeed, the refilling of the inner region is due only to the polariton tunneling across the soliton; hence, the achievable polariton density in between the solitons decreases as the polariton decay rate increases; the equilibrium between the density pressure and the soliton repulsion, therefore, takes place for a smaller separation distance. In Fig. 6, the error bars also result from the small breathing, responsible for small oscillations of the solitons in the propagation range where the separation distance is averaged. For our sample, the polariton lifetime is $10 \mathrm{ps}$, which corresponds to a linewidth of $0.07 \mathrm{meV}$. In Fig. 6, the blue dot illustrates the experimental result and conditions, i.e., the average equilibrium separation distance for the decay rate of our microcavity, in good agreement with the theory.

\section{CONCLUSION}

In conclusion, we implemented a new technique to imprint phase patterns on a quasiresonantly pumped polariton fluid by engineering the driving field with an SLM. By imprinting the solitons and exploiting the optical bistability, we enhanced their propagation distance for more than one order of magnitude with respect to the previous works. This technique permits one not only to artificially form dark solitons at will, but also to study the soliton interaction: Remarkably, we have observed an unconventional binding mechanism, leading to the formation of a new kind of darksoliton molecule in a local nonlinear medium. Moreover, we demonstrated that the characteristic separation distance between the solitons of the molecule is governed by the 
driven-dissipative nature of the polariton fluid. This result opens unprecedented possibilities to control and manipulate collective excitations such as dark solitons enabling the generation of multiple-soliton patterns and the study of their mutual interactions as well as their robustness against modulational instabilities. More generally, the developed method opens the way to a quantitative study of quantum turbulence phenomena in quantum fluids of light.

\section{ACKNOWLEDGMENTS}

This work has received funding from the French ANR grants (C-FLigHT 138678 and "Quantum Fluids of Light" ANR-16-CE30-0021) and from the European Unions Horizon 2020 Research and Innovation Program under Grant Agreement No. 820392 (PhoQuS). Q. G. and A. B. thank the Institut Universitaire de France (IUF) for support.

[1] M. Segev and G. Stegeman, Self-Trapping of Optical Beams: Spatial Solitons, Phys. Today 51, No. 8, 42 (1998).

[2] Y. S. Kivshar and B. Luther-Davies, Dark Optical Solitons: Physics and Applications, Phys. Rep. 298, 81 (1998).

[3] D. J. Frantzeskakis, Dark Solitons in Atomic Bose-Einstein Condensates: From Theory to Experiments, J. Phys. A 43, 213001 (2010).

[4] W. Zhao and E. Bourkoff, Interactions between Dark Solitons, Opt. Lett. 14, 1371 (1989).

[5] G. A. Swartzlander, D. R. Andersen, J. J. Regan, H. Yin, and A. E. Kaplan, Spatial Dark-Soliton Stripes and Grids in Self-Defocusing Materials, Phys. Rev. Lett. 66, 1583 (1991).

[6] J. Denschlag, Generating Solitons by Phase Engineering of a Bose-Einstein Condensate, Science 287, 97 (2000).

[7] D. Foursa and P. Emplit, Investigation of Black-Gray Soliton Interaction, Phys. Rev. Lett. 77, 4011 (1996).

[8] M. Chen, M. A. Tsankov, J. M. Nash, and C. E. Patton, Microwave Magnetic-Envelope Dark Solitons in Yttrium Iron Garnet Thin Films, Phys. Rev. Lett. 70, 1707 (1993).

[9] T. Shinjo, T. Okuno, R. Hassdorf, K. Shigeto, and T. Ono, Magnetic Vortex Core Observation in Circular Dots of Permalloy, Science 289, 930 (2000).

[10] G. A. Williams, Vortex-Loop Phase Transitions in Liquid Helium, Cosmic Strings, and High- Tc Superconductors, Phys. Rev. Lett. 82, 1201 (1999).

[11] A. M. Weiner, J. P. Heritage, R. J. Hawkins, R. N. Thurston, E. M. Kirschner, D. E. Leaird, and W. J. Tomlinson, Experimental Observation of the Fundamental Dark Soliton in Optical Fibers, Phys. Rev. Lett. 61, 2445 (1988).

[12] S. Stellmer, C. Becker, P. Soltan-Panahi, E.-M. Richter, S. Dörscher, M. Baumert, J. Kronjäger, K. Bongs, and K. Sengstock, Collisions of Dark Solitons in Elongated BoseEinstein Condensates, Phys. Rev. Lett. 101, 120406 (2008).

[13] A. Chabchoub, O. Kimmoun, H. Branger, N. Hoffmann, D. Proment, M. Onorato, and N. Akhmediev, Experimental Observation of Dark Solitons on the Surface of Water, Phys. Rev. Lett. 110, 124101 (2013).
[14] M. Stratmann, T. Pagel, and F. Mitschke, Experimental Observation of Temporal Soliton Molecules, Phys. Rev. Lett. 95, 143902 (2005).

[15] E. A. Ostrovskaya, Y. S. Kivshar, Z. Chen, and M. Segev, Interaction between Vector Solitons and Solitonic Gluons, Opt. Lett. 24, 327 (1999).

[16] N. I. Nikolov, D. Neshev, W. Królikowski, O. Bang, J. J. Rasmussen, and P. L. Christiansen, Attraction of Nonlocal Dark Optical Solitons, Opt. Lett. 29, 286 (2004).

[17] Q. Kong, Q. Wang, O. Bang, and W. Krolikowski, Analytical Theory for the Dark-Soliton Interaction in Nonlocal Nonlinear Materials with an Arbitrary Degree of Nonlocality, Phys. Rev. A 82, 013826 (2010).

[18] A. Dreischuh, D. N. Neshev, D. E. Petersen, O. Bang, and W. Krolikowski, Observation of Attraction between Dark Solitons, Phys. Rev. Lett. 96, 043901 (2006).

[19] J. C. Martinez, Creation of Dark-Soliton Atoms, Europhys. Lett. 96, 14007 (2011).

[20] C. Ciuti, V. Savona, C. Piermarocchi, A. Quattropani, and P. Schwendimann, Role of the Exchange of Carriers in Elastic Exciton-Exciton Scattering in Quantum Wells, Phys. Rev. B 58, 7926 (1998).

[21] A. Amo, S. Pigeon, D. Sanvitto, V. G. Sala, R. Hivet, I. Carusotto, F. Pisanello, G. Lemenager, R. Houdre, E. Giacobino, C. Ciuti, and A. Bramati, Polariton Superfluids Reveal Quantum Hydrodynamic Solitons, Science 332, 1167 (2011).

[22] G. Grosso, G. Nardin, F. Morier-Genoud, Y. Léger, and B. Deveaud-Plédran, Soliton Instabilities and Vortex Street Formation in a Polariton Quantum Fluid, Phys. Rev. Lett. 107, 245301 (2011).

[23] P. M. Walker, L. Tinkler, B. Royall, D. V. Skryabin, I. Farrer, D. A. Ritchie, M. S. Skolnick, and D. N. Krizhanovskii, Dark Solitons in High Velocity Waveguide Polariton Fluids, Phys. Rev. Lett. 119, 097403 (2017).

[24] R. Hivet, H. Flayac, D. D. Solnyshkov, D. Tanese, T. Boulier, D. Andreoli, E. Giacobino, J. Bloch, A. Bramati, G. Malpuech et al., Half-Solitons in a Polariton Quantum Fluid Behave like Magnetic Monopoles, Nat. Phys. 8, 724 (2012).

[25] H. Flayac, D. D. Solnyshkov, and G. Malpuech, Separation and Acceleration of Magnetic Monopole Analogs in Semiconductor Microcavities, New J. Phys. 14, 085018 (2012).

[26] X. Ma, O. A. Egorov, and S. Schumacher, Creation and Manipulation of Stable Dark Solitons and Vortices in Microcavity Polariton Condensates, Phys. Rev. Lett. 118, 157401 (2017).

[27] Y. Xue, Y. Jiang, G. Wang, R. Wang, S. Feng, and M. Matuszewski, Creation of Stable Dark and Anti-dark Solitons in Polariton Dyad, Opt. Express 26, 6267 (2018).

[28] L. A. Smirnov, D. A. Smirnova, E. A. Ostrovskaya, and Y. S. Kivshar, Dynamics and Stability of Dark Solitons in Exciton-Polariton Condensates, Phys. Rev. B 89, 235310 (2014).

[29] A. M. Kamchatnov and L. P. Pitaevskii, Stabilization of Solitons Generated by a Supersonic Flow of Bose-Einstein Condensate past an Obstacle, Phys. Rev. Lett. 100, 160402 (2008).

[30] S. V. Koniakhin, O. Bleu, D. D. Stupin, S. Pigeon, A. Maitre, F. Claude, G. Lerario, Q. Glorieux, A. Bramati, 
D. Solnyshkov, and G. Malpuech, Stationary Quantum Vortex Street in a Driven-Dissipative Quantum Fluid of Light, Phys. Rev. Lett. 123, 215301 (2019).

[31] S. V. Koniakhin, O. Bleu, G. Malpuech, and D. D. Solnyshkov, $2 d$ Quantum Turbulence in a Polariton Quantum Fluid, Chaos Solitons Fractals 132, 109574 (2020).

[32] T. Simula, M. J. Davis, and K. Helmerson, Emergence of Order from Turbulence in an Isolated Planar Superfluid, Phys. Rev. Lett. 113, 165302 (2014).

[33] G. Gauthier, M. T. Reeves, X. Yu, A. S. Bradley, M. A. Baker, T. A. Bell, H. Rubinsztein-Dunlop, M. J. Davis, and T. W. Neely, Giant Vortex Clusters in a Two-Dimensional Quantum Fluid, Science 364, 1264 (2019).

[34] S. P. Johnstone, A. J. Groszek, P. T. Starkey, C. J. Billington, T. P. Simula, and K. Helmerson, Evolution of Large-Scale Flow from Turbulence in a Two-Dimensional Superfluid, Science 364, 1267 (2019).

[35] I. Carusotto and C. Ciuti, Quantum Fluids of Light, Rev. Mod. Phys. 85, 299 (2013).

[36] A. Amo, J. Lefrère, S. Pigeon, C. Adrados, C. Ciuti, I. Carusotto, R. Houdré, E. Giacobino, and A. Bramati, Superfluidity of Polaritons in Semiconductor Microcavities, Nat. Phys. 5, 805 (2009).

[37] S. Pigeon, I. Carusotto, and C. Ciuti, Hydrodynamic Nucleation of Vortices and Solitons in a Resonantly Excited Polariton Superfluid, Phys. Rev. B 83, 144513 (2011).

[38] D. Sanvitto, S. Pigeon, A. Amo, D. Ballarini, M. De Giorgi, I. Carusotto, R. Hivet, F. Pisanello, V. G. Sala, P. S. S. Guimaraes, R. Houdré, E. Giacobino, C. Ciuti, A. Bramati, and G. Gigli, All-Optical Control of the Quantum Flow of a Polariton Condensate, Nat. Photonics 5, 610 (2011).

[39] T. Boulier, S. Pigeon, E. Cancellieri, P. Robin, E. Giacobino, Q. Glorieux, and A. Bramati, Coherent Merging of
Counterpropagating Exciton-Polariton Superfluids, Phys. Rev. B 98, 024503 (2018).

[40] T. Boulier, E. Cancellieri, N. D. Sangouard, Q. Glorieux, A. V. Kavokin, D. M. Whittaker, E. Giacobino, and A. Bramati, Injection of Orbital Angular Momentum and Storage of Quantized Vortices in Polariton Superfluids, Phys. Rev. Lett. 116, 116402 (2016).

[41] G. Nardin, G. Grosso, Y. Léger, B. Pitka, F. Morier-Genoud, and B. Deveaud-Plédran, Hydrodynamic Nucleation of Quantized Vortex Pairs in a Polariton Quantum Fluid, Nat. Phys. 7, 635 (2011).

[42] A. Baas, J. P. Karr, H. Eleuch, and E. Giacobino, Optical Bistability in Semiconductor Microcavities, Phys. Rev. A 69, 023809 (2004).

[43] I. Carusotto and C. Ciuti, Probing Microcavity Polariton Superfluidity through Resonant Rayleigh Scattering, Phys. Rev. Lett. 93, 166401 (2004).

[44] S. Pigeon and A. Bramati, Sustained Propagation and Control of Topological Excitations in Polariton Superfluid, New J. Phys. 19, 095004 (2017).

[45] G. Lerario, A. Maître, R. Boddeda, Q. Glorieux, E. Giacobino, S. Pigeon, and A. Bramati, Vortex-Stream Generation and Enhanced Propagation in a Polariton Superfluid, Phys. Rev. Research 2, 023049 (2020).

[46] G. Lerario, S. V. Koniakhin, A. Maître, D. Solnyshkov, A. Zilio, Q. Glorieux, G. Malpuech, E. Giacobino, S. Pigeon, and A. Bramati, Parallel Dark Soliton Pair in a Bistable $2 d$ Exciton-Polariton Superfluid, arXiv:2003.11408.

[47] L. P. Pitaevskii and S. Stringari, Bose-Einstein Condensation (Oxford University Press, 2003), ISBN: 9780198507192.

[48] D. J. Frantzeskakis, Dark Solitons in Atomic Bose-Einstein Condensates: From Theory to Experiments, J. Phys. A 43, 213001 (2010). 